\title{
Specific PIWI-interacting small noncoding RNA expression patterns in pulmonary tuberculosis patients
}

\author{
Xing Zhang ${ }^{\ddagger}, 1$, Zi Liang ${ }^{\ddagger}, 1$, Yunshan Zhang ${ }^{\ddagger}, 1$, Min Zhu ${ }^{1}$, Yueping Zhu², Sumei Li ${ }^{3}$, Weifeng

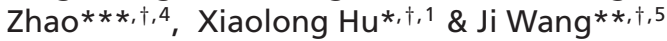 \\ ${ }^{1}$ School of Biology \& Basic Medical Science, Soochow University, Suzhou 215123, PR China \\ ${ }^{2}$ Department of Infectious Disease, The Fifth People's Hospital of Suzhou, Suzhou 215000, PR China \\ ${ }^{3}$ Department of Tuberculosis, The Fifth People's Hospital of Suzhou, Suzhou 215000, PR China \\ ${ }^{4}$ Department of Infectious Disease, First Affiliated Hospital of Soochow University, Suzhou 215006, PR China \\ ${ }^{5}$ Department of Oncology, The Second Affiliated Hospital of Soochow University, Suzhou 215004, PR China \\ †Postal address: 199 No. Renai Road, Suzhou Industrial Park, Suzhou City, Jiangsu Province 215123, PR China \\ *Author for correspondence: Tel.: +86-521-65880183; Fax: +86-521-65880183; xlhu2013@suda.edu.cn \\ **Author for correspondence: royking_333@163.com \\ ***Author for correspondence: zhaoweifeng@suda.edu.cn \\ $¥$ Authors contributed equally
}

\begin{abstract}
Aim: PIWl-interacting RNAs (piRNAs) play crucial roles in germline development and carcinogenesis. The expression patterns of piRNAs in pulmonary tuberculosis (PTB) are still unclear. Materials \& methods: Small RNA sequencing was applied to investigate peripheral blood piRNA expression patterns in PTB patients and healthy individuals. Results: A total of 428 upregulated and 349 downregulated piRNAs were identified from PTB patients. Target genes of dysregulated piRNAs were mainly involved in transcription and protein binding. Dysregulated piRNAs were enriched in many pathways related with immunity. Many target genes were regulated by the same piRNAs. Nucleotide bias of these piRNAs showed that piRNAs in peripheral blood may be formed from the primary biogenesis pathway. Conclusion: Findings demonstrated that the PIWI-piRNA pathway is active in human peripheral blood, where it may represent a new player in the PTB pathogenesis.
\end{abstract}

First draft submitted: 4 September 2018; Accepted for publication: 25 October 2019; Published online: 22 November 2019

Keywords: expression pattern $\bullet$ healthy individuals $\bullet$ peripheral blood $\bullet$ piRNA clusters $\bullet$ piRNA $\bullet$ PTB patients $\bullet$ pulmonary tuberculosis $\bullet$ regulatory interaction $\bullet$ small RNA sequencing • target genes

Tuberculosis (TB) is an infectious disease caused by Mycobacterium tuberculosis. This mycobacterium not only affects the lungs but also affects other sites [1]. Only 5-15\% of people infected with $M$. tuberculosis will develop TB during their lifetime [2]. This disease has remained one of the major threats to health [3]. TB pathogenesis and the immune response to $M$. tuberculosis infection have not been completely elucidated. Therefore, understanding the components of the host response will help us to a better understanding of the pathogenesis of TB in humans, and to find novel approaches to prevention and therapy of this infectivity disease [4-6].

Noncoding small RNAs, regulatory RNAs, regulate the expression of target genes at the transcriptional and translational levels [7-9]. miRNAs have emerged as important regulators of the immune response [10-14]. miRNA expression level is differentially regulated in macrophages following mycobacterial infection. Expression of miRNAs in macrophages can be induced by extracted mycobacterial cell-wall components [15]. A total of 15 miRNAs were expressed uniquely in latent TB infection with miRNA microarray [16]. miRNA (miR)-133a inhibits collagen degradation by downregulating matrix metalloproteinase- 9 expression to attenuate the destructive effects of spinal TB on intervertebral discs [17]. The miR-26a/KLF4 and CREB-C/EBP $\beta$ signaling pathways play important roles in regulating the survival of $M$. tuberculosis in macrophages [18]. The presences of circulating extracellular RNA (ExRNAs) from the tissues injury have the potential to be as biomarker for a great variety of medical conditions [19]. Our previous studies have shown that the expression levels of six serum miRNAs in the peripheral blood of patients 
with pulmonary tuberculosis (PTB) are significantly different from those in healthy individuals. Logistic regression analysis of six serum miRNAs can be used for diagnosis of PTB [20]. Comprehensively understand the relationship between noncoding small RNAs and PTB will help us to better understand the PTB pathogenesis.

Compared with miRNAs, piRNAs are a novel type of noncoding small RNAs that interact with a subset of Argonaute proteins, and are a less-investigated type of small RNA [21,22]. This type of noncoding small RNA was first found in mammalian systems in 2006 [23-25]. They are 26-31 nucleotides (nt) long and are clearly distinct from miRNAs that are 21-24 nt [26]. PiRNAs have been studied extensively in Drosophila [27,28], mice [29], Caenorhabditis elegans [30,31], zebrafish [32], Aedes aegypti [33], Tribolium castaneum [34] and Cubitus interruptus [35]. Many roles of piRNAs were reported, such as maintaining the integrity of germline DNA, heterochromatin formation, sex determination and germline development [36,37]. However, most of piRNAs identified involved in inside of germline, and the roles played by piRNAs outside of germline are still poorly investigated [38]. However, there is an increasing number of reports about piRNA identification and function outside the germline, such as breast cancer [39,40], hepatocellular carcinoma [41], Alzheimer's disease [42,43], renal cell carcinoma [44], pancreatic cancer [45], bladder cancer [46] and gastric cancer [47]. In addition, piRNAs are stably expressed in human serum and plasma samples, which can serve as valuable blood-based biomarkers for disease detection and monitoring [48]. Recent findings on piRNAs suggest that these novel noncoding RNAs are crucial regulators that contribute to the progression of human diseases.

In this study, small RNA sequencing showed that piRNAs were aberrantly expressed in the peripheral blood of PTB patients and healthy individuals. Many dysregulated piRNAs were found and these aberrantly expressed piRNAs may be associated with the pathogensis of PTB. This study provided the first evidence of piRNAs in the peripheral blood of PTB patients, which will provide the foundation to determine the regulatory role of piRNAs in PTB.

\section{Materials \& methods \\ Patients}

All the PTB patients and healthy individuals were recruited from the First People's Hospital of Zhangiiagang, Jiangsu Province, China. PTB patients were diagnosed based on clinical manifestations. Healthy individuals had no family history of hereditary diseases or low immune function. The characters for PTB patients and healthy individuals were described in our published paper [49,50]. Three PTB patients and three healthy individuals were selected for detecting small RNAs with small RNA sequencing. Other 20 normal healthy people as control group and 20 PTB patients as test group were recruited for further validation.

\section{Small RNA sequencing}

Peripheral blood was collected from PTB patients and healthy individuals and the total RNAs was extracted from these samples according to our published paper $[49,50]$. All RNA samples were submitted to Shanghai Oebiotech (China) and sequencing was performed on an Illumina Hiseq 4000.

\section{Bioinformatics analysis}

Obtained raw data/reads were filtered remove the low-quality reads, reads with $5^{\prime}$-primer contaminants and poly (A). Reads without $3^{\prime}$-adapter and insert tags, and reads shorter than $15 \mathrm{nt}$ and longer than $41 \mathrm{nt}$ from raw data were filtered to obtain clean reads. Samll RNAs were aligned and subjected to BLAST (v2.2.28+) search against Rfam (version 10.0) (http://www.sanger.ac.uk/software/Rfam) with E-value $\leq 0.01$, miRBase database (http: //www.mirbase.org/) and piRBase (http://www.regulatoryrna.org/database/piRNA/). Through the above several methods to filter, obtained clean reads were aligned with the known piRNA from the piRBase with Bowtie software without mismatches. The novel piRNAs were predicted with Piano (http://ento.njau.edu.cn/Piano.html) according to the structure and sequence characteristics of the interaction between transposon and piRNAs [51]. Transcript per million (TPM) was used to calculate the expression levels of these piRNAs [52]. piRNAs were considered differentially expressed (DE) when showing absolute fold change $\geq 2$ with false discovery rate (FDR) $\leq 0.05$, as determined by DESeq method (http://bioconductor.org/packages/release/bioc/html/DESeq.html). The biological processes associated with mRNAs targeted by the DE piRNAs were predicted with Miranda algorithm (singleresidue-pair match scores $\geq 150$, specific Gibbs free energy $(\Delta \mathrm{G}) \leq-30 \mathrm{kcal} / \mathrm{mol}$ and Demand strict $5^{\prime}$ seed pairing). Gene Ontology (GO) enrichment and Kyoto Encyclopedia of Genes and Genomes (KEGG) pathway enrichment analysis of DE piRNA target genes were based on hypergeometric distribution $(\mathrm{p} \leq 0.05)$. 


\section{Western blotting}

To estimate the expression levels of PIWIL2 and PIWIL4 proteins in the peripheral blood, three PTB patients and three healthy individuals were included. Total protein was extracted from the peripheral blood with a whole blood protein extraction kit. $30 \mu \mathrm{g}$ total proteins were run on a $12 \%$ polyacrylamide gel. The antibodies included rabbit polyclonal anti-PIWIL2 and anti-PIWIL4 (1: 1000; Abcam, MA, USA). Next, goat anti-rabbit horseradish-peroxidase-conjugated secondary antibody (1: 2000; Abcam) was used and then visualized using enhanced chemiluminescence (Millipore, MA, USA). GAPDH (Abcam, MA, USA) was selected as the protein internal reference control.

\section{piRNA-mRNA network construction}

To explore the relationship between piRNAs and target mRNAs in PTB progression, the coexpression networks were constructed based on the correlation analysis between the DE piRNAs and target mRNAs. One network was constructed with upregulated piRNAs and downregulated mRNAs, and another with downregulated piRNAs and upregulated mRNAs. Many interaction pairs were identified, and only the top 100 interacting pairs were shown according to the lowest p-value.

\section{piRNA clusters analysis}

Many piRNAs derived from particular genomic sites are termed as piRNA clusters. the presence of piRNA clusters in the peripheral blood of PTBs were searched with proTRAC software with a scanning window of length $5 \mathrm{~kb}$ and a window shift of $1 \mathrm{~kb}$. Significant $(\mathrm{p} \leq 0.01)$ hit density was calculated based on observed hit distribution.

\section{Real-time PCR}

To examine the dysregulation of piRNAs indentified from the PTB patients from RNA sequencing, three upregulated piRNAs (piRNA-1007467, piR-hsa-1344 and piR-hsa-1944) and three downregulated piRNAs (piR-hsa32157, piRNA-276256 and piRNA-1088346) were selected and validated with real-time PCR. According to the Bulge-loop $^{T M}$ miRNA validation method, the forward primers of six DE piRNAs were designed based on the mature sequence of these piRNAs, and the reverse primers of them were designed according to the published method [53]. U6 gene was used to normalize the expression level of these piRNAs, respectively. All of the primers were shown in the Supplementary Table 1. Total RNAs extraction and real-time PCR validation were conducted according to the methods described in our previous reports [49]. Relative expression levels of piRNAs were calculated by the $2^{-\Delta \Delta C t}$ method. Statistical analysis was performed using T-test.

\section{Results}

PIWIL proteins profiles in the peripheral blood of PTB patients \& healthy individuals

The PIWI protein subfamily is essential for piRNAs biogenesis and the expression of PIWILs is related with the presence of active piRNAs pathways. In our previous study, PIWIL2 and PIWIL4 genes were expressed in the peripheral blood of PTB patients and healthy individuals by transcriptome sequencing (Figure 1A). To investigate the active piRNA pathways in the peripheral blood of PTB patients, the expression of two human PIWIL proteins (PIWIL2 and PIWIL4) were surveyed in PTB and healthy samples by Western blotting. Two human PIWI orthologs, PIWIL2 and PIWIL4, were present in PTB patients and healthy individuals (Figure 1B). Except these, the related genes (EXD1, FKBP6, HENMT1, MAEL, MOV1OL1, PLD6, TDRD1, TDRD12, TDRD9 and TDRKH) with piRNA metabolic processes were checked from the transcriptome sequencing, and these genes were expressed in peripheral blood (Figure 1C). Hence, we suggest that active piRNA pathways appear in the peripheral blood of PTB patients and may play some key regulatory roles in PTB pathogenesis.

\section{Small RNA sequencing reveals piRNA in peripheral blood of PTB patients \& healthy individuals}

To understand the piRNA expression patterns in the peripheral blood of PTB patients and healthy individuals, small RNA sequencing was applied to investigate the small RNA expression pattern. The peripheral blood of PTB patients and healthy individuals were used for total RNAs extraction, and the length of 15-41 nt small RNA were sequenced. Small RNA profiling of mapped reads revealed two distinct peaks, one at the 21-23 nt position, corresponding to miRNA in size, and another at the 31-33 nt position, corresponding to piRNAs in size (Figure 2A). From each sample, 22,672,995-26,645,820 reads aligned to the human genome sequence dataset (GRCh38.p7) were obtained, which included miRNA (0.61\%), rRNA (0.03\%), tRNA (0.01\%), snRNA (0.03\%), cis-reg (0.03\%), 
(A)

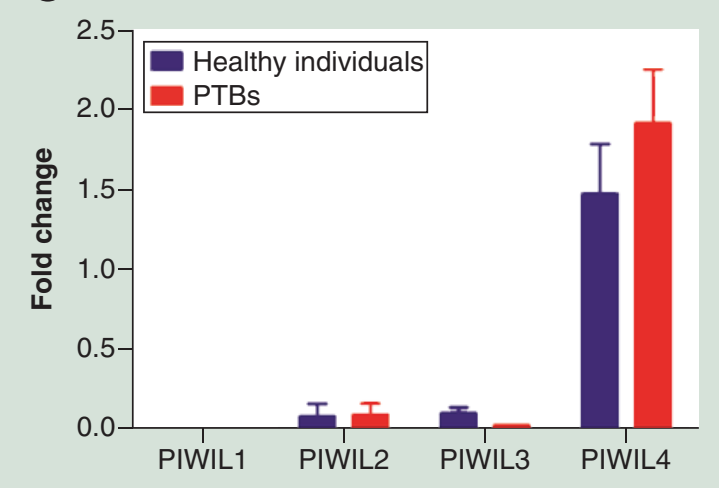

(C)

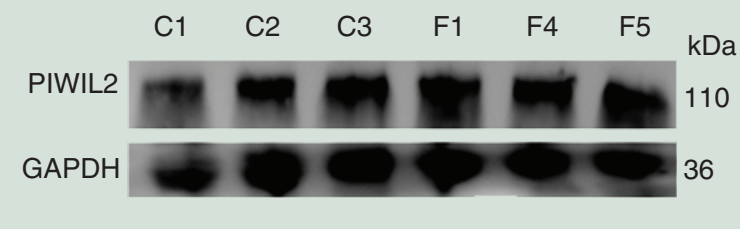

(B)

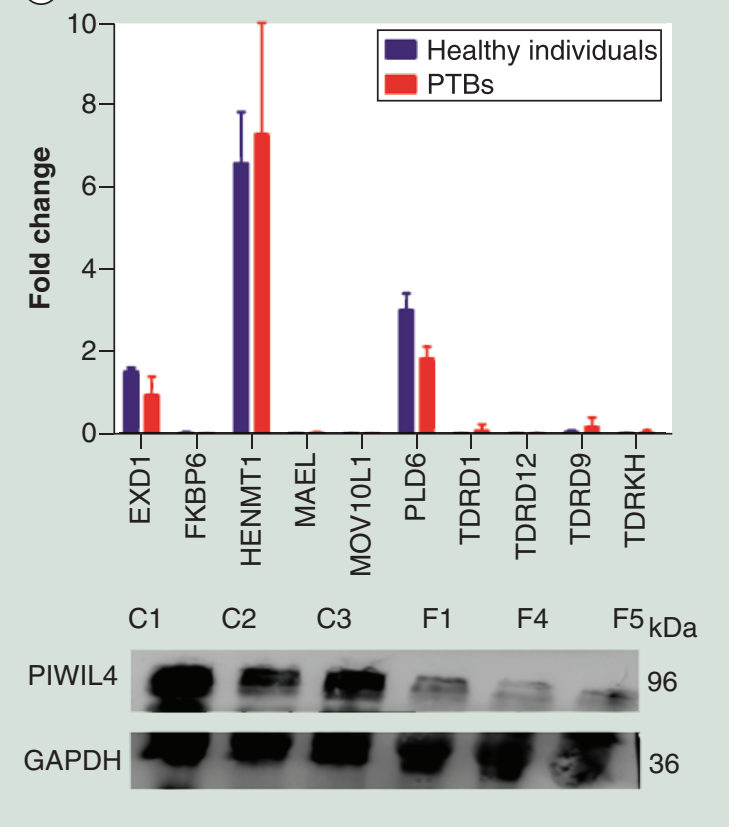

Figure 1. Expression of PIWIL mRNAs and proteins in the peripheral blood of pulmonary tuberculosis patients and healthy individuals. (A) Expression of PIWIL1, PIWIL2, PIWIL3 and PIWIL4 genes in the peripheral blood of pulmonary tuberculosis (PTB) patients and healthy individuals with transcriptome sequencing. PIWIL1, PIWIL2, PIWIL3 and PIWIL4 represent PIWI-like RNA-mediated gene silencing 1, 2, 3 and 4. (B) PIWIL2 and PIWIL4 proteins were detected by Western blotting in the peripheral blood of three PTB patients and three healthy individuals. GAPDH was used as the reference control. C1, C2 and C3 represent healthy individuals, and F1, F4 and F5 represent PTB patients. (C) Expression of genes related to piRNA metabolism with transcriptome sequencing. PTB: Pulmonary tuberculosis.

others (0.07\%) and unannotated (98.69\%) (Figure 2B). In addition to the small RNAs aligned to known sncRNA genes, the remaining reads were aligned to piRBase (http://www.regulatoryrna.org/database/piRNA/). The piRBase currently contains 7.7 billion piRNAs sequences originally discovered from nine species, such as humans, mice, fruitfly, zebrafish and other species (Figure 2C), which can be aligned to the GRCh38.p7 genome without mismatches. Through the repeated sequences filtered, obtained clean reads were aligned with the known piRNA from the piRBase with Bowtie software without mismatches. Nearly 500 known piRNAs identified from each sample. Piano (http://ento.njau.edu.cn/Piano.html) was used to predict piRNAs. A total of 454,320 piRNAs were predicted and 39481 piRNAs were expressed in peripheral blood cells (Supplementary Table 2). These finding demonstrated that piRNAs were present in the peripheral blood of PTB patients and healthy individuals, which also indicated that piRNAs are highly conserved across species and present in the somatic tissues.

piRNAs are DE in the peripheral blood of PTB patients \& healthy individuals

To investigate DE piRNAs in the peripheral blood of PTB patients and healthy individuals, the identified piRNAs (known and novel piRNAs) were subjected to differential expression analysis with the DESeq method. Expression of piRNAs in PTB patients and healthy individuals was measured based on TPM (transcript per million), which indicated no abnormal expression in the six samples (Figure 3A). The number and distribution of piRNAs from the different samples were displayed in the volcano plot (Figure 3B and 3C). Hierarchical clustering revealed a clear segregation of the samples from PTB patients from healthy individuals (Figure 3D). Total 777 DE piRNAs were identified from the PTB patients, including 428 upregulated and 349 downregulated piRNAs (fold change $>2$, and FDR $<0.05)$. A total of 142 and $192 \mathrm{DE}$ piRNAs were only specifically expressed in healthy individuals and PTB patients, respectively (Figure 4A). To understand the authenticity of these dysregulated piRNAs from the sequencing data, a set of piRNAs (piRNA-1007467, piR-hsa-1344, piR-hsa-1944, piR-hsa-32157, piRNA-276256 


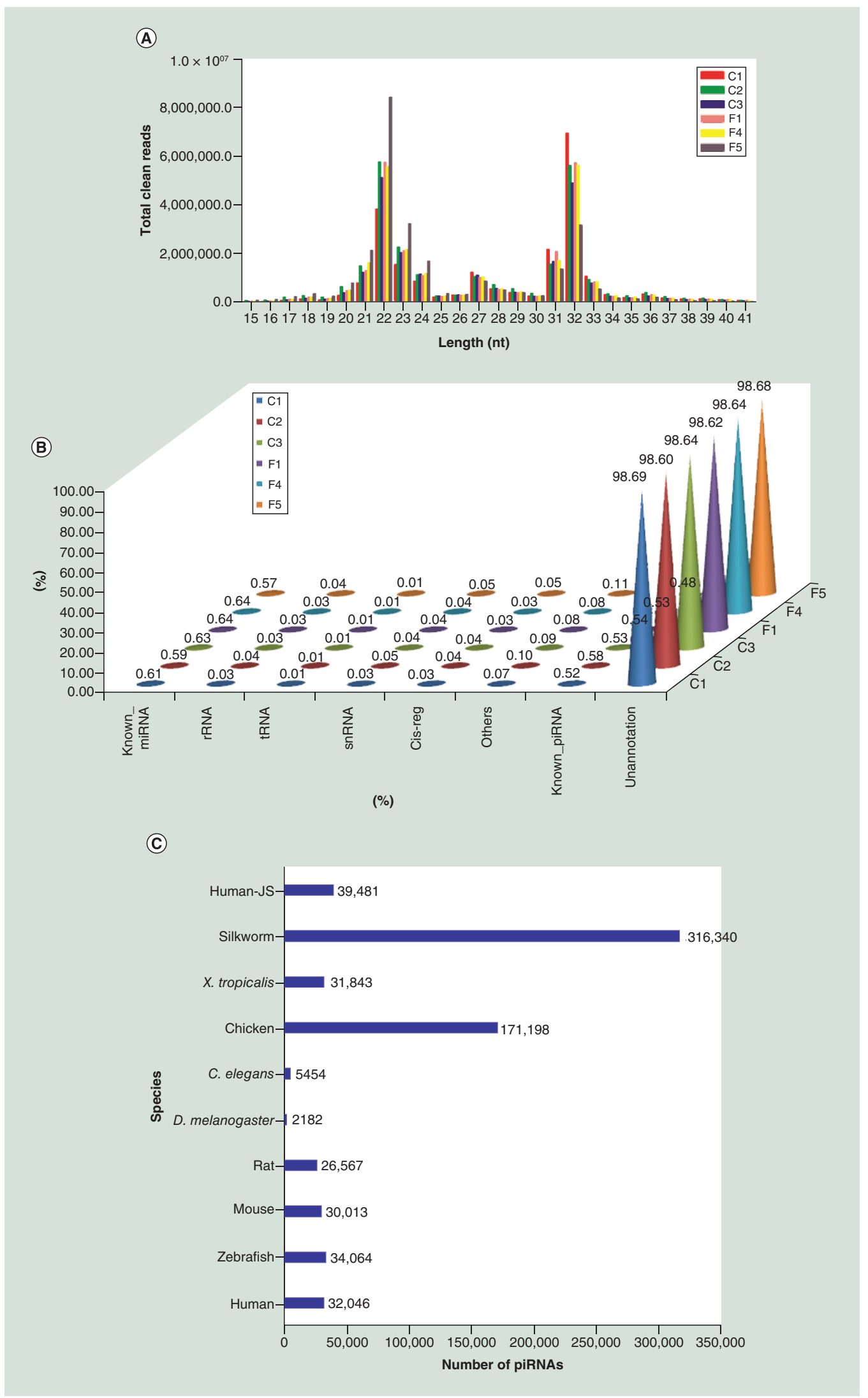

Figure 2. Characterization of PIWI-interacting RNAs present in the peripheral blood of pulmonary tuberculosis patients and healthy individuals. (A) Length distribution of the small RNAs identified with small RNA sqeuencing. (B) Category of small RNA with uniq-clean reads from the small RNA sequencing. (C) Number of piRNAs from different species (humans, silkworm, $X$. tropicalis, chicken, C. elegans, D. melanogaster, rat, mouse, zebrafish) and the data were collected from the piRBase (http://www.regulatoryrna.org/database/piRNA/). piRNA: PIWI-interacting RNA. 
(A)

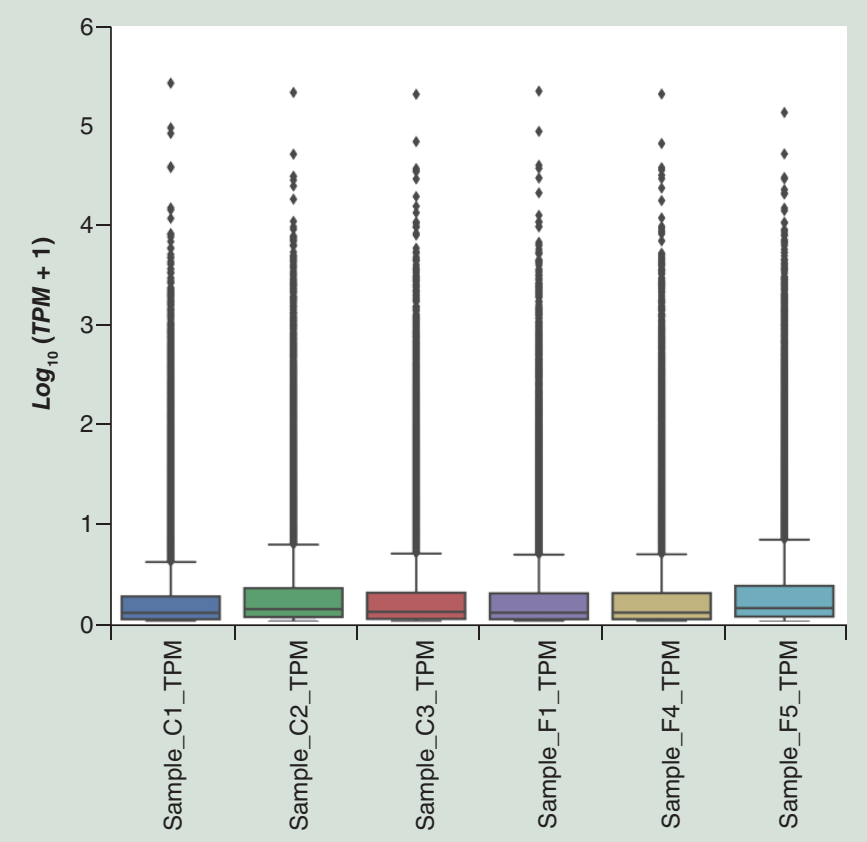

(B)

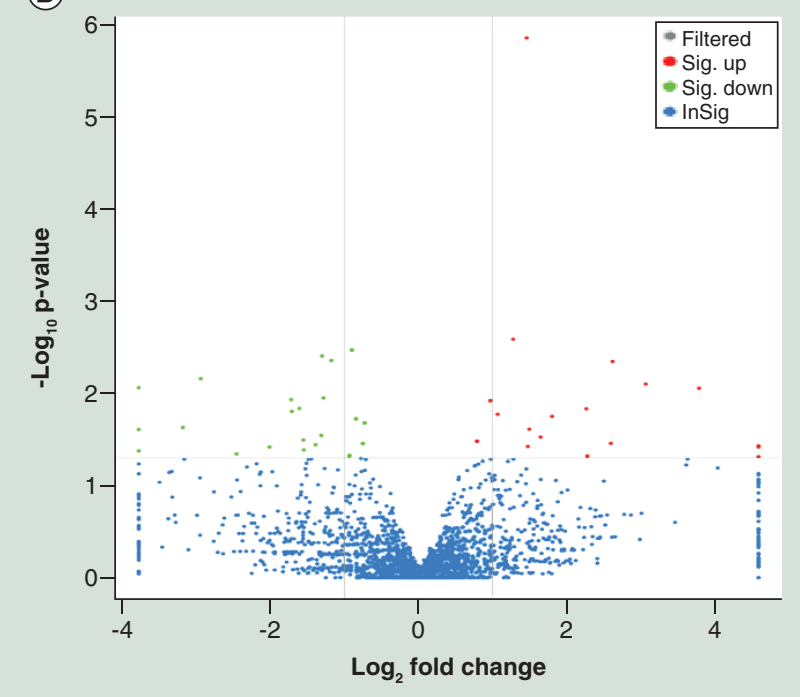

(C)

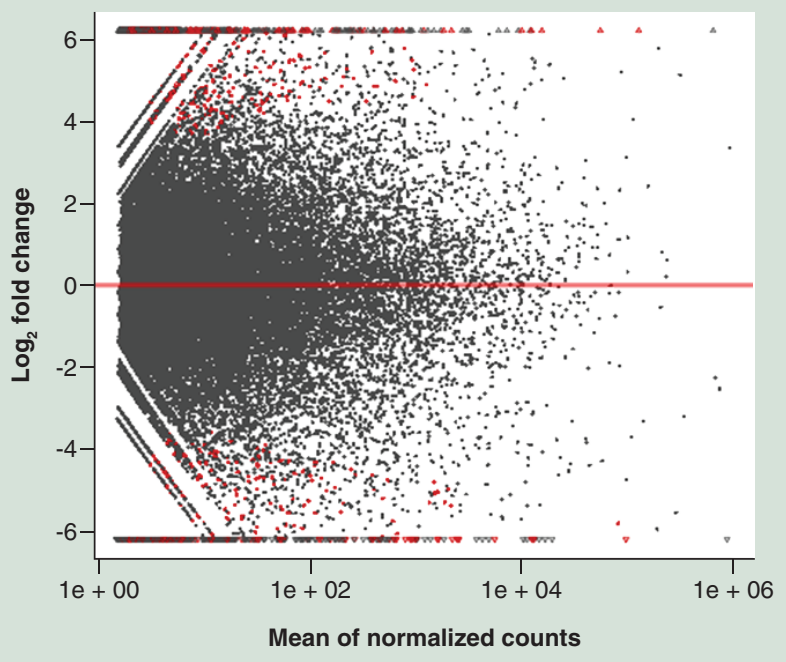

(D)

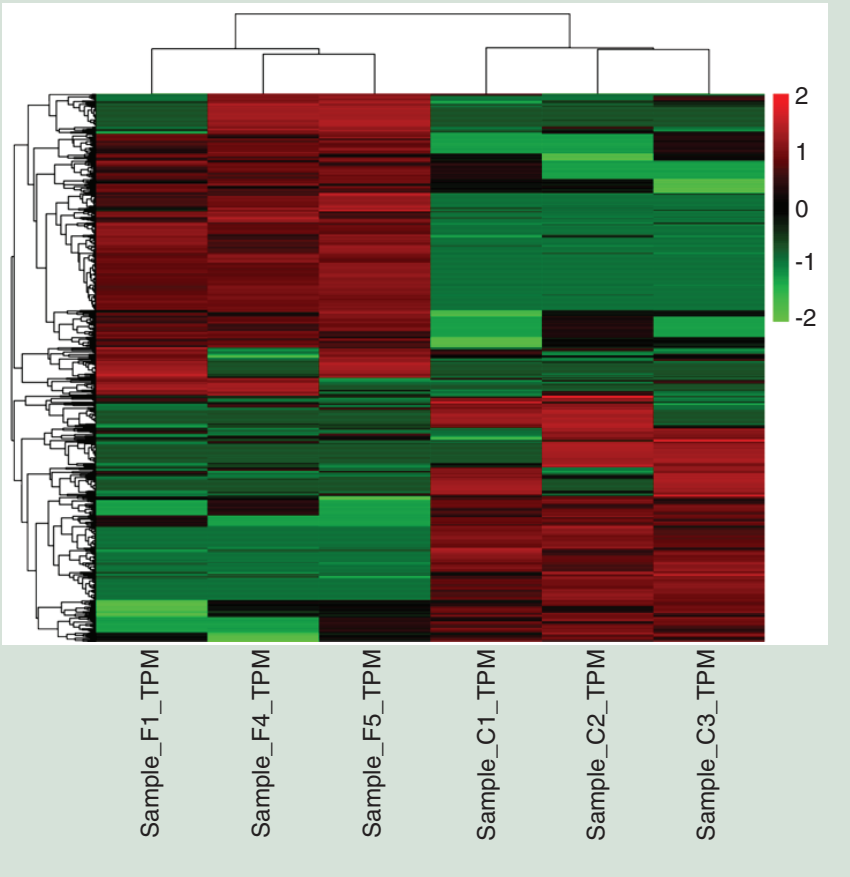

Figure 3. Expression of PIWI-interacting RNAs in the peripheral blood of pulmonary tuberculosis patients and healthy individuals. (A) Box plot showing quality assessment of PIWI-interacting RNA (piRNA) data after filtering. (B) MA plot of differentially expressed piRNAs in peripheral blood of pulmonary tuberculosis (PTB) patients and healthy individuals. (C) Volcano plots showing differentially expressed piRNAs in peripheral blood of PTB patients and healthy individuals. The vertical lines correspond to a 2.0-fold increase and decrease, respectively, and the horizontal line represents a p-value of 0.05 . The red point in the plot represents the significantly differentially expressed piRNAs. (D) Heatmaps representing mean centered and normalized data relative to differentially expressed piRNAs in the peripheral blood of three PTBs and three healthy individuals with average fold change $>2 ; \mathrm{p}<0.05$ and FDR $<0.05$.

and piRNA-1088346) was investigated using real-time PCR (Figure 4B). The results from the real-time PCR were consistent with the sequencing data, which indicated that the sequencing data were accurate and reproducible. 
(A)

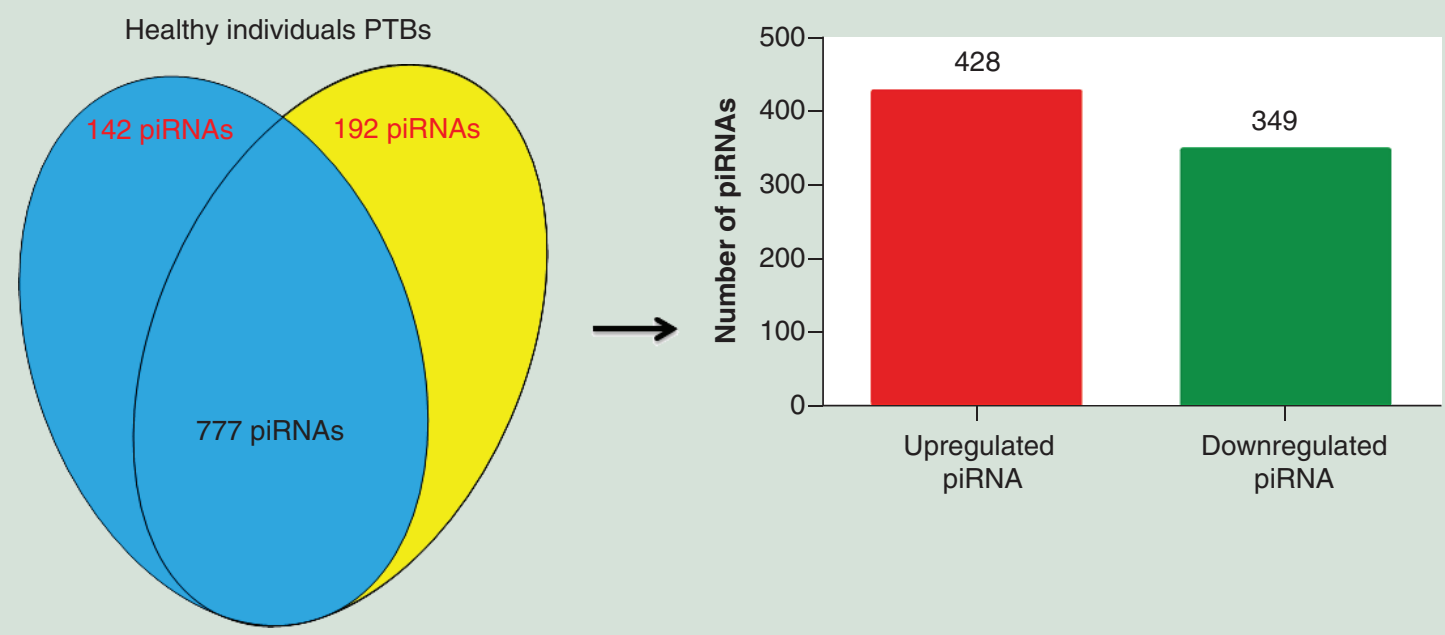

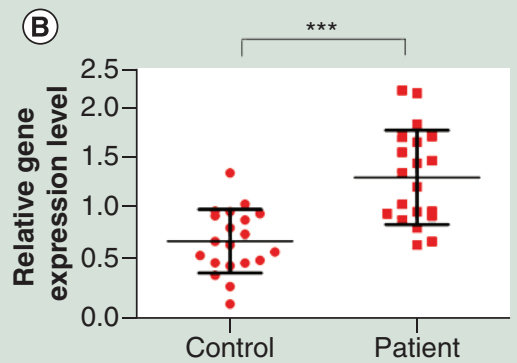

piRNA-1007467

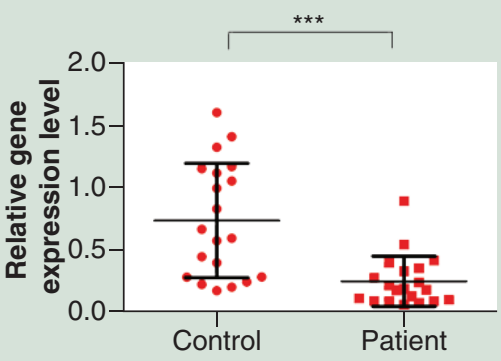

piR-hsa-32157

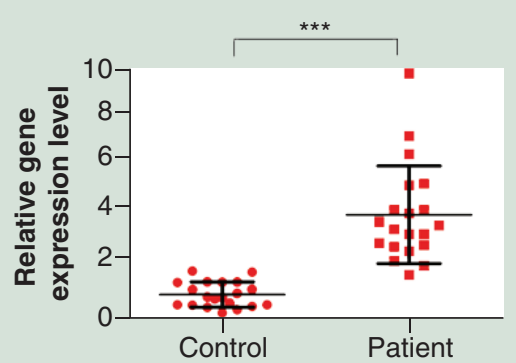

piR-hsa-1344

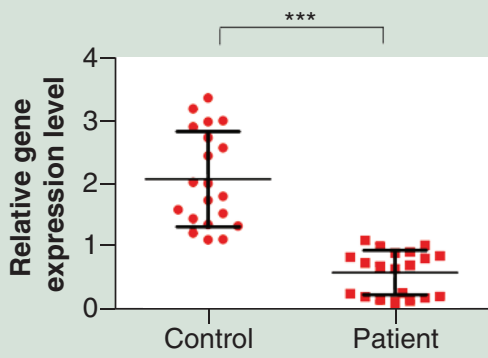

piRNA-276256

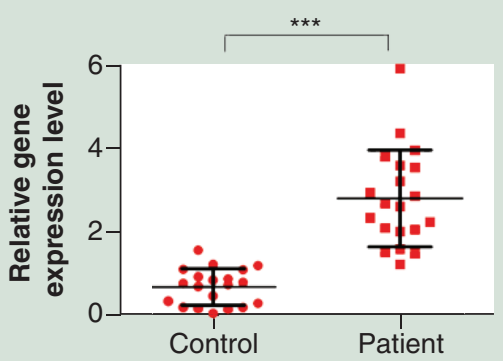

piR-hsa-1944

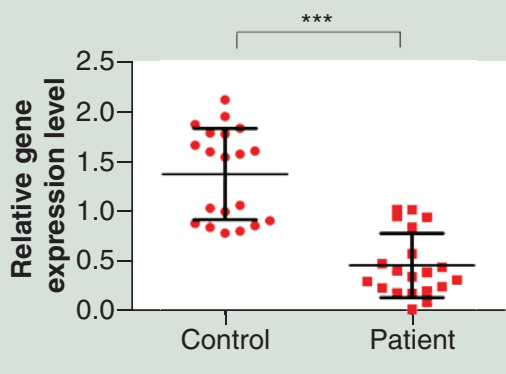

piRNA-1088346

Figure 4. Identification of differentially expressed PIWI-interacting RNAs in peripheral blood of pulmonary tuberculosis patients and healthy individuals. (A) PiRNA Venn diagram for pulmonary tuberculosis patients versus healthy individuals and the differentially expressed piRNAs in peripheral blood of PTB patients and healthy individuals. (B) Validation of piRNAs expression levels with real-time PCR. Six differentially expressed piRNAs (piRNA-1007467, piR-hsa-1344, piR-hsa-1944, piR-hsa-32157, piRNA-276256 and piRNA-1088346) from the small RNA sequencing were further validated with real-time PCR. The expression levels of six piRNAs were examined in 20 pulmonary tuberculosis patients and 20 healthy individual. U6 was used as the reference gene. Relative expression levels of piRNAs were calculated by the $2^{-\Delta \Delta C t}$ method. Statistical analysis was performed using t-test. ${ }^{* *} \mathrm{p}<0.001$.

piRNA: PIWI-interacting RNAs; PTB: Pulmonary tuberculosis.

\section{Target prediction of DE piRNAs}

To understand the potential roles of these DE piRNAs, mRNAs targeted by the DE piRNAs were predicted with Miranda algorithm. All the target genes were derived from our whole transcriptome data. Many key genes involved in important signaling pathways were targeted by the dysregulated piRNAs, such as pathways in cancer, 
regulation of actin cytoskeleton, proteoglycans in cancer, rap1 signaling pathway and cGMP-PKG signaling pathway (Supplementary Table 3).

\section{Functional annotation of DE piRNAs}

To understand the roles of DE piRNAs in PTB patients, GO annotation and KEGG pathway analysis were used. If the GO function set was significantly enriched in the DE piRNAs, a hypergeometric distribution test was used to calculate the p-value of them, which was then corrected by multiple Benjamini-Hochberg tests.

According to the routine GO classification algorithms, the top 10 GO classification of biological process (BP), cell components (CC) and molecular function (MF) were shown according to the enriched dysregulated piRNAs derived from the gene annotation $(\mathrm{p}<0.05)$. The top three GO processes of upregulated/downregulated piRNAs included transcription, regulation of transcription and signal transduction in the BP subgroup. Cytoplasm, nucleus and plasma membrane were the top three processes in the CC subgroup. Protein binding, metal ion binding, and ATP binding were the top three processes in the MF subgroup (Figure 5A \& B).

Three hundred and one KEGG pathways (multiple test of Benjamini \& Hochberg) were identified among the dysregulated piRNAs. Enrichment of the signaling pathways of these dysregulated piRNAs showed that upregulated piRNAs were enriched in key pathways, such as pathway in cancer, regulation of actin cytoskeleton and Rap1 signaling pathway (Figure 6A). Downregulated piRNAs were enriched important pathways, such as pathway in cancer, regulation of actin cytoskeleton and Wnt signaling pathway (Figure 6B). These results support that the piRNAs represent a novel type of regulators in the pathogenesis of PTB.

\section{piRNA-mRNA interaction networks construction}

To know the regulatory mechanism of piRNAs in PTB, the targets of DE piRNAs were predicted with the Miranda algorithm. piRNAs have a similar function to miRNAs to degrade specific mRNAs. Therefore, the upregulated piRNAs (downregulated piRNAs)/downregulated (upregulated) mRNAs of piRNA targets were selected to construct the networks. Because of the reverse regulation of piRNAs to the targets, one network was constructed with upregulated piRNAs and downregulated mRNAs, and another with downregulated piRNAs and upregulated mRNAs. Many interaction pairs were identified, and only the top 100 interacting pairs were shown according to the p-value. From the correlation analysis of upregulated piRNAs and downregulated mRNAs network, piRNA881565, piRNA-489848, piRNA-1869760, piRNA-784007 and piRNA-1503138 regulated 34, 32, 28, 21 and 18 mRNA targets, respectively (Figure 7A). piRNA-1216758, piRNA-1577991, piRNA-866055, piRNA-1403892 and piRNA-1 154851 regulated 41,32, 27, 20 and 13 mRNA targets, respectively (Figure 7B). One mRNA can be regulated by several piRNAs. The results showed that many targets interacted with piRNAs, which indicated dysregulated expression of piRNAs in the peripheral blood of PTB patients.

\section{piRNA clusters in PTBs}

To reveal the genomic piRNA clusters in PTB, proTRAC software was used. We found 24 highly dense piRNA clusters including $115 \mathrm{DE}$ piRNAs from the genome of PTB patients. High cluster density was observed on chromosomes of 16 and 19, it harbored four and two piRNA clusters with 22 and 15 piRNAs in PTB. No clusters were detected on chromosomes 9, 13, 14, 21, 22, X and Y (Figure 8A). Nucleotides bias of these piRNAs identified in piRNA clusters was investigated, which showed that piRNAs encoded from the plus strand had the highest bias for $1 \mathrm{U}$ as compared with the minus strand, while a higher bias for $10 \mathrm{~A}$ was observed in the minus strand (Figure $8 \mathrm{~B}$ ). Thus, we speculated that piRNAs in peripheral blood may be mainly generated from the primary biogenesis mechanism.

\section{Discussion}

piRNAs, a novel noncoding RNAs, are abundant and extensively expressed in germline and gonads, but little is known about their expression patterns and roles in the peripheral blood of patients with infectious diseases, especially PTB. Four PIWI proteins in piRNAs biogenesis, PIWIL1, PIWIL2, PIWIL3 and PIWIL4 were encoded by human genome [54,55]. piRNAs can bind to PIWI proteins to form complexes to induce target gene silencing effect [56,57]. A variety roles of piRNAs are reported, such as, regulation of transposable elements in the germline [58], regulation of histone modifications at the binding site [59] and post-transcriptional regulation of gene expression [60]. In the present study, piRNA expression patterns were first investigated in the peripheral blood of PTB patients and healthy individuals and 39481 expressed piRNAs were identified. We found that PIWIL2 and PIWIL4 proteins 


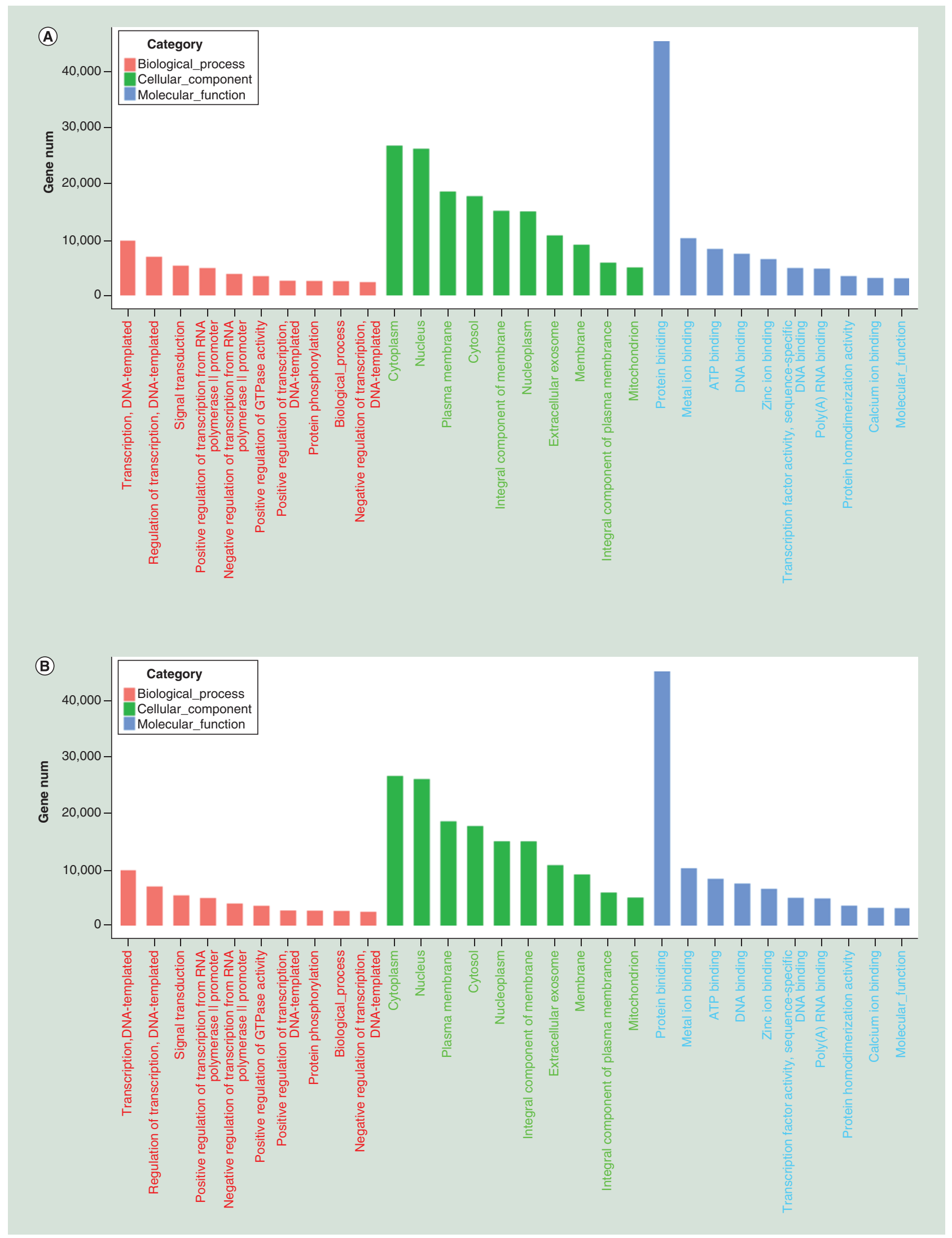

Figure 5. Gene ontology annotation analysis with the targets of differentially expressed PIWI-interacting RNAs. (A) GO annotation analysis of the targets of upregulated PIWl-interacting RNAs. According to the enrichment, the GO terms were divided into biological process, cell components and molecular function subgroups. (B) GO annotation analysis of the targets of downregulated PIWI-interacting RNAs. According to the enrichment, the GO terms were divided into biological process, cell components and molecular function subgroups. 
(A)

KEGG enrichment top 20

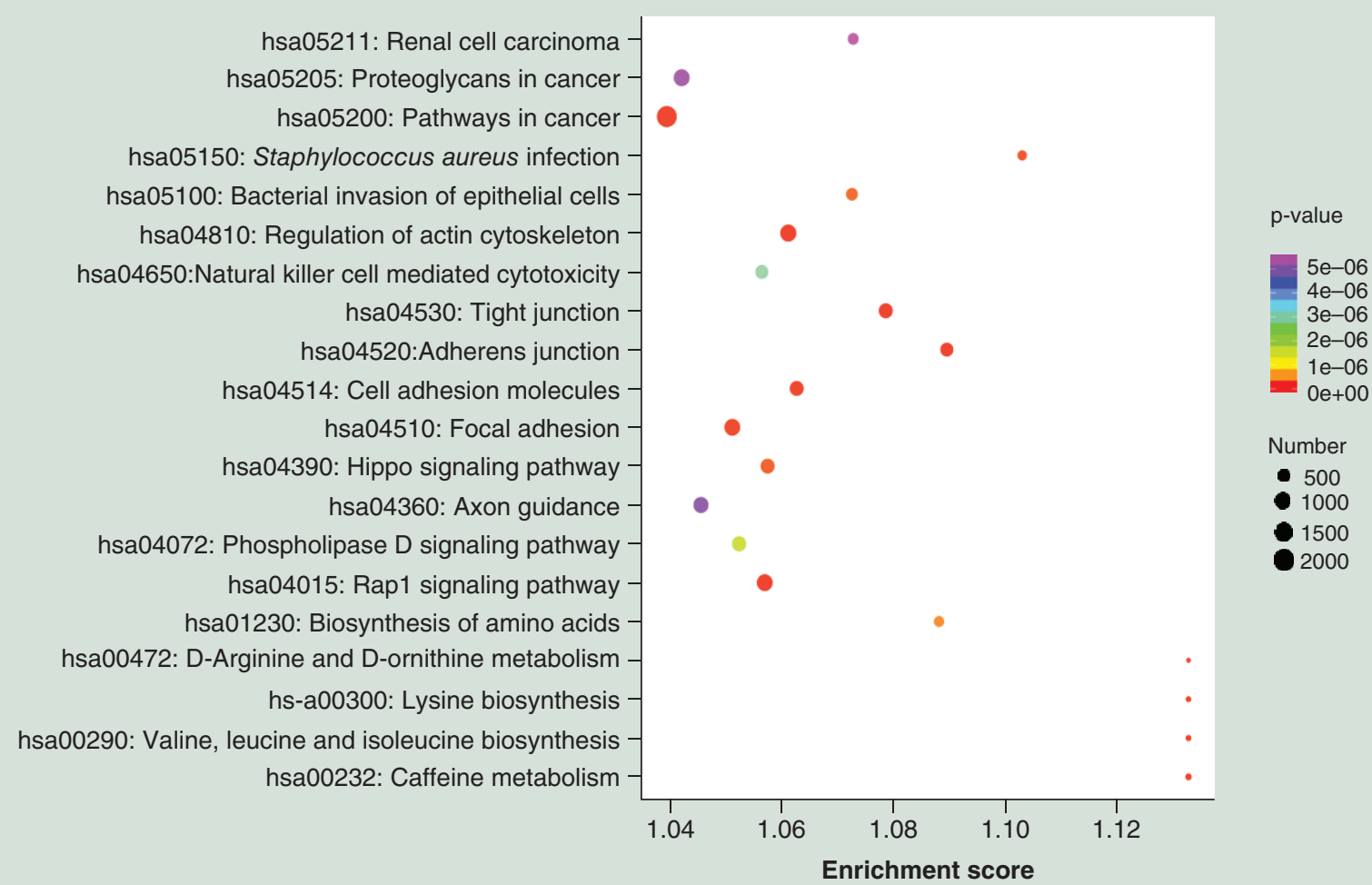

(B)

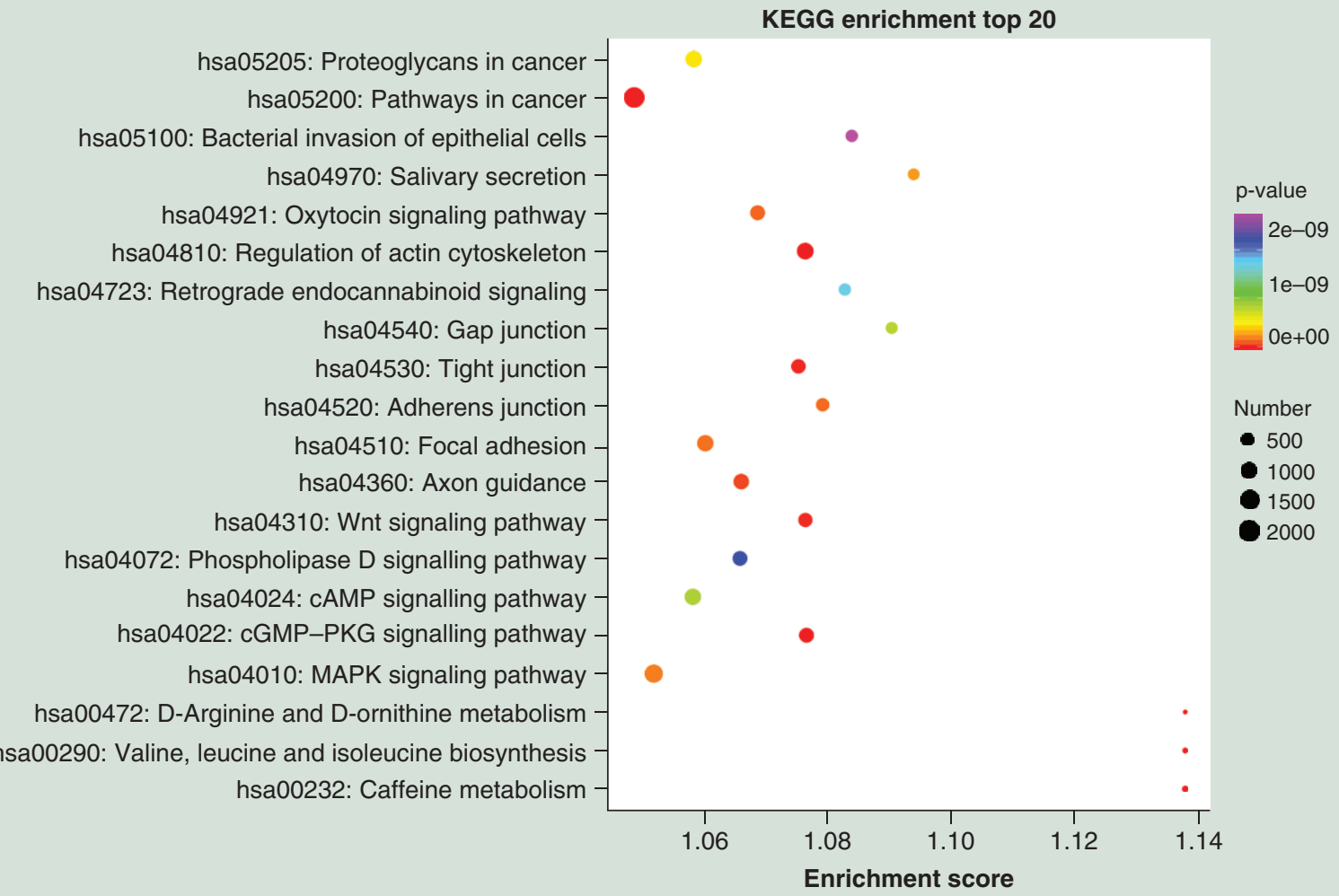

Figure 6. Kyoto Encyclopedia of Genes and Genomes enrichment analysis with the targets of differentially expressed PIWI-interacting RNAs. (A) KEGG enrichment analysis of the targets of upregulated piRNAs. (B) KEGG enrichment analysis of the targets of downregulated piRNAs. Graph size represents the numbers of genes and graph color represents the p-value. According to the multiple test of Benjamini \& Hochberg, the top 20 enriched KEGG pathways were used to shown on the enrichment picture. 
(A)

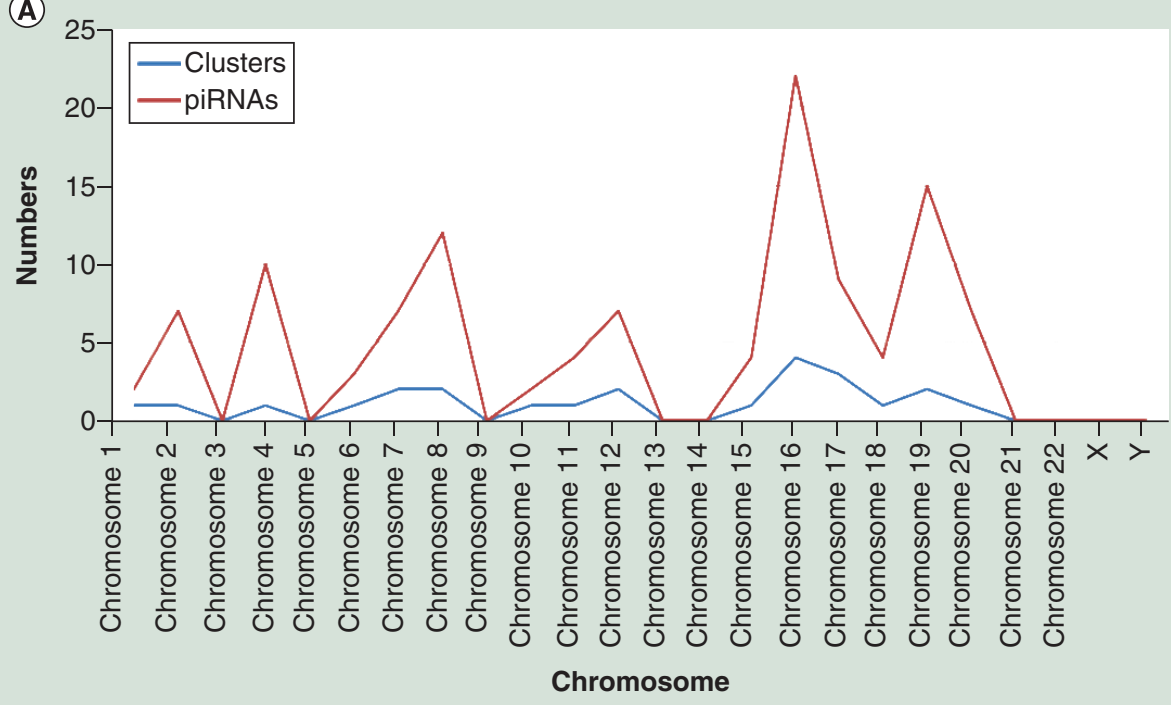

(B)

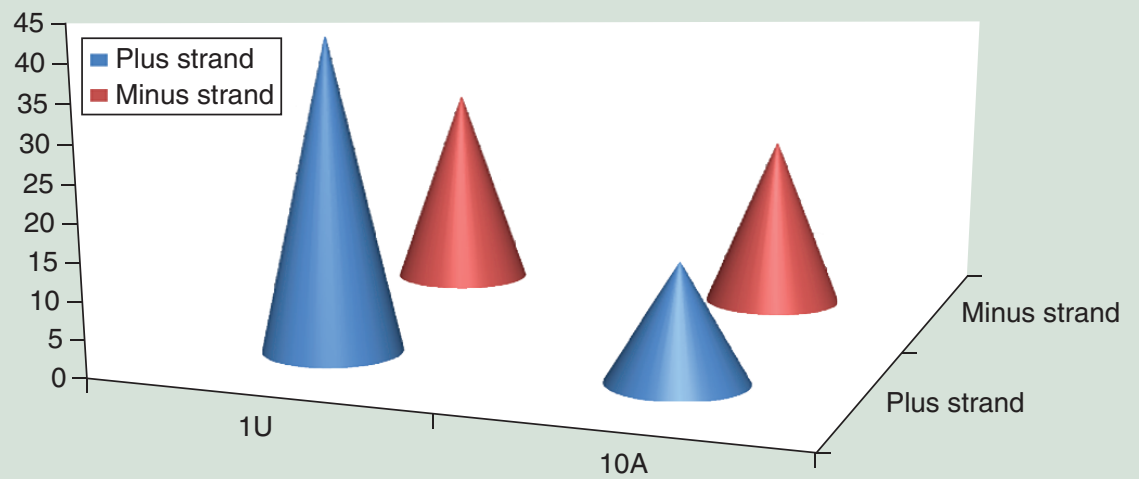

Figure 7. PIWI-interacting RNA clusters distribution on the chromosomes and in the peripheral blood of pulmonary tuberculosis patients. (A) Number of piRNA clusters and piRNAs across various chromosomes. (B) $1 \mathrm{U}$ and $10 \mathrm{~A}$ bias among piRNAs encoded from plus and minus of the identified piRNA clusters from the pulmonary tuberculosis patients.

were expressed in the peripheral blood. The genes related to piRNA metabolism were also expressed in peripheral blood. Expression of PIWI proteins, piRNA-related genes and piRNAs in the peripheral blood indicates that the PIWI-piRNA system is working, and this system is not only present in the germline, but is also active in the peripheral blood.

The expression profiles of piRNAs were investigated to gain insights into their possible functions in PTB pathogenesis. Screening of patients with PTB and healthy individuals $(\geq$ twofold change and FDR $<0.05)$ identified 777 piRNAs with significant levels of differential expression, with 428 significantly unregulated and 349 significantly downregulated. This clearly demonstrates piRNA dysregulation in PTB patients, indicating that many genes involved in piRNA pathways are also dysregulated in PTB. Dysregulated piRNAs in the peripheral blood have also been reported in gastric cancer patients, and expression of piR-651 and piR-823 is significantly lower than in healthy individuals [47]. piRNAs are stably expressed in human serum or plasma samples [48]. Multiple sequences consistent with piRNA and snoRNA species have been identified in human plasma [61]. Therefore, we propose that these dysregulated piRNAs in the peripheral blood of PTB patients may be associated with the development and pathogenesis of diseases with different roles played in the germline. It is known that the role of piRNA machinery is to mediate epigenetic silencing of target diverse mRNAs, and act as siRNAs to degrade specific mRNAs [62]. Maternal mRNAs are destabilized in the early Drosophila embryo, and they are targeted by abundant piRNAs and their deadenylation depends on the piRNA pathway [63]. Functional analysis of RNA targeted by dysregulated 


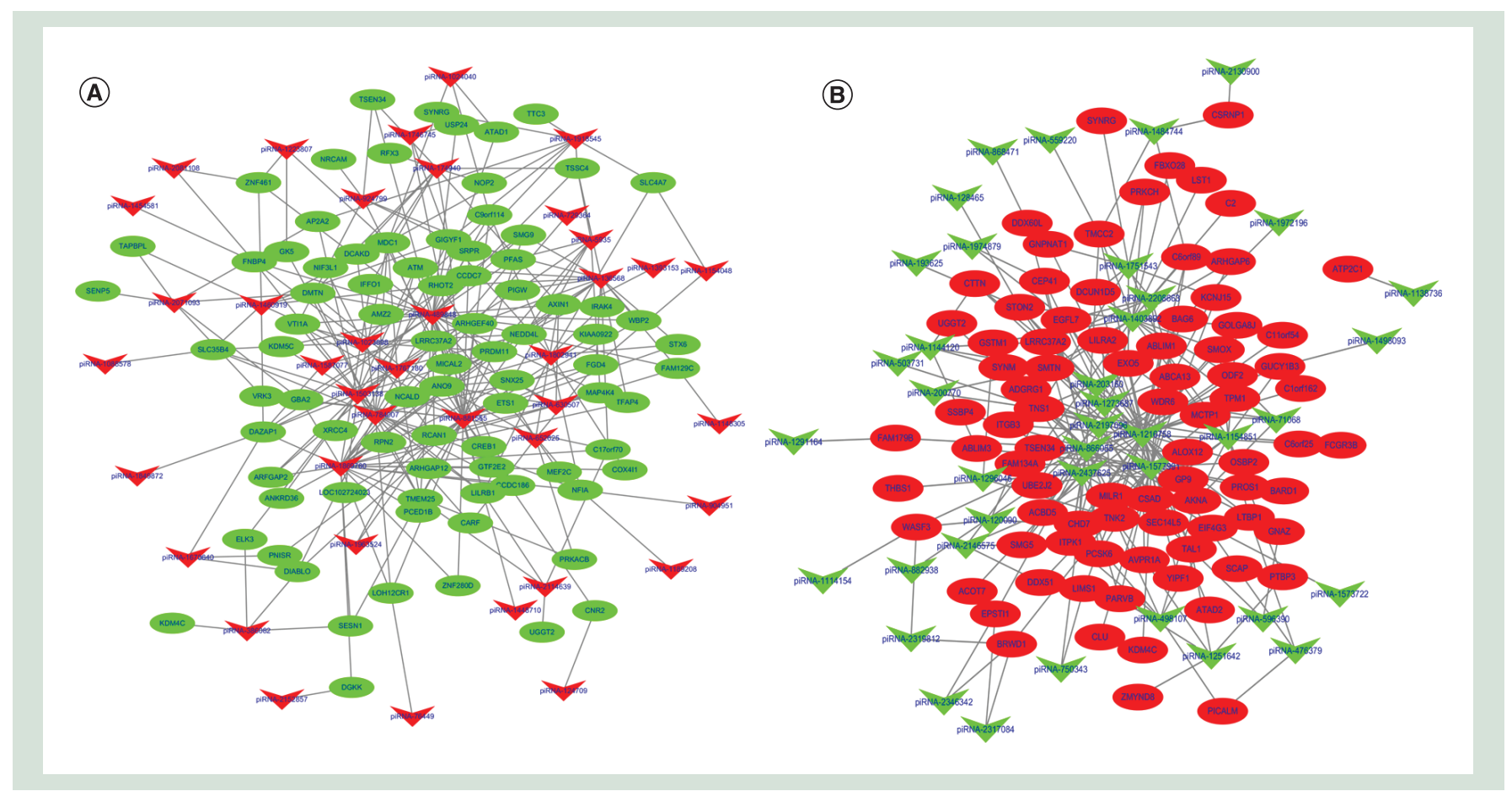

Figure 8. Correlation analysis of PIWI-interacting RNAs and mRNAs in peripheral blood of pulmonary tuberculosis patients. (A) Correlation analysis of upregulated piRNAs and downregulated mRNAs with top 100 piRNAs according to p-value. Red triangular represents piRNA, green ellipse represents mRNA. (B) Correlation analysis of downregulated piRNAs and upregulated mRNAs with top 100 piRNAs according to $p$ value. Green triangular represents piRNA, red ellipse represents mRNA.

piRNAs expressed in PTB patients reveals a significant correlation with several major important pathways that are dysregulated in PTB, including signaling pathway in cancer, Rap1, Wnt and cGMP-PKG. The piRNA targets include protein binding, metal ion binding, ATP binding, DNA binding, zinc ion binding and transcript factor activity, which are all known to be active in the process of PTB pathogenesis.

Two piRNA biogenesis pathways are in germline cells, which are the primary maturation pathway and a secondary pathway (also termed 'ping-pong' cycle) [64]. The primary maturation pathway of piRNAs shows a strong preference for $\mathrm{U}$ at the $5^{\prime}$ end and no nucleotide bias at position 10 . The secondary pathway show a bias for $\mathrm{A}$ at position 10 and no $5^{\prime}$ end bias [65]. The primary pathway is in the germline and ovarian somatic follicle cells, and the secondary pathway is only in active only in the germline $[66,67]$. The piRNAs identified from the peripheral blood of PTB patients showed a strong preference for $\mathrm{U}$ at the $5^{\prime}$ end and no nucleotide bias at position 10, suggesting that their synthesis is likely to occur through the primary maturation pathway.

Mammalian embryonic piRNAs are involved in regulating endogenous gene expression [68], and we investigated significant negative correlations between piRNAs targeting mRNAs and piRNAs. To understand the potential molecular mechanisms of piRNAs in the peripheral blood of PTB patients, the targets (mRNA) of DE piRNAs were predicted from our whole transcriptome data, and many important genes with immune roles were identified. According to the negative regulation of piRNAs to the targets, the correlation analysis network between piRNAs and mRNAs was constructed. Here, we propose that many genes were targeted by piRNAs and which may through the siRNA-like function of the piRNA machinery in the peripheral blood of PTBs.

\section{Future perspective}

This study is believed to be the first to evaluate comprehensively the differential expression of piRNAs between PTB patients and healthy individuals by small RNA sequencing. The piRNA expression patterns and pathways analysis presented here offer insights for the elucidation of detailed functions of piRNAs in PTB pathogenesis. In the future, all of these DE piRNAs and target genes of piRNAs will be validated with other methods. The MFs of 
these dysregulated piRNAs will be investigated in specific immune cells. Whether piRNAs can be used as biomarker for early diagnosis of PTB, this will be done in large number of clinical samples.

\section{Summary points}

- Dysregulated piRNAs were first indentified from the peripheral blood of pulmonary tuberculosis (PTB) patients.

- A total of 428 upregulated and 349 downregulated piRNAs were identified from PTB patients.

- Target genes of piRNAs were mainly involved in transcription and protein binding.

- Dysregulated piRNAs were enriched in many pathways related with immunity.

- piRNAs in peripheral blood may be formed from the primary biogenesis pathway.

- piRNAs regulate the target genes by siRNA-like mechanism.

- piRNAs may be as an important noncoding RNAs in pathogenesis of PTB.

- piRNAs were first identified in the peripheral blood of PTB patients.

Financial \& competing interests disclosure

This work was supported by Natural Science Foundation of Jiangsu Province (grant no. BK20150300) and the Natural Science Foundation of the Jiangsu Higher Education Institutions of China (grant no. 19KJB320005). The authors have no other relevant affiliations or financial involvement with any organization or entity with a financial interest in or financial conflict with the subject matter or materials discussed in the manuscript apart from those disclosed.

No writing assistance was utilized in the production of this manuscript.

\section{Open access}

This work is licensed under the Attribution-NonCommercial-NoDerivatives 4.0 Unported License. To view a copy of this license, visit http://creativecommons.org/licenses/by-nc-nd/4.0/

\section{References}

Papers of special note have been highlighted as: $\bullet$ of interest; $\bullet \bullet$ of considerable interest

1. Smith I. Mycobacterium tuberculosis pathogenesis and molecular determinants of virulence. Clin. Microbiol. Rev. 16(3), $463-496$ (2003).

2. Zumla A, George A, Sharma V et al. The WHO 2014 global tuberculosis report-further to go. Lancet. Global Health 3(1), e10-e12 (2015).

3. Raviglione M, Sulis G. Tuberculosis 2015: burden, challenges and strategy for control and elimination. Infect. Dis. Rep. 8(2), 33-37 (2016).

4. Banerjee SK, Kumar M, Alokam R et al. Targeting multiple response regulators of Mycobacterium tuberculosis augments the host immune response to infection. Sci. Rep.UK 6, 25851 (2016).

-. Targeting multiple response regulator may be developed as a novel antimycobacterial agents against drug-resistant Mycobacterium tuberculosis.

5. Fletcher HA. Profiling the host immune response to tuberculosis vaccines. Vaccine 33(40), 5313-5315 (2015).

6. Schluger NW, Rom WN. The host immune response to tuberculosis. Am. J. Resp. Crit. Care 157(3), 679-691 (1998).

7. Li J, Zhang YJ, Li DM et al. Small non-coding RNAs transfer through mammalian placenta and directly regulate fetal gene expression. Protein Cell 6(6), 391-396 (2015).

8. Aalto AP, Pasquinelli AE. Small non-coding RNAs mount a silent revolution in gene expression. Curr. Opin. Cell Biol. 24(3), 333-340 (2012).

9. Shimoni Y, Friedlander G, Hetzroni G et al. Regulation of gene expression by small non-coding RNAs: a quantitative view. Mol. Syst. Biol. 3 (2007).

10. Ha TY. The role of microRNAs in regulatory t cells and in the immune response. Immune Netw. 11(1), 11-41 (2011).

11. Zhang Y, Li YK. MicroRNAs in the regulation of immune response against infections. J. Zhejiang Univ. Sci. B 14(1), 1-7 (2013).

12. Bettencourt P, Pires D, Anes E. Immunomodulating microRNAs of mycobacterial infections. Tuberculosis 97, 1-7 (2016).

13. Yang T, Ge B. miRNAs in immune responses to Mycobacterium tuberculosis infection. Cancer Lett. 431, 22-30 (2018).

14. Yi Z, Gao K, Li R, Fu Y. Changed immune and miRNA response in RAW264.7 cells infected with cell wall deficient Mycobacterium tuberculosis. Int. J. Mol. Med. 41(5), 2885-2892 (2018).

15. Abdalla AE, Duan X, Deng W, Zeng J, Xie J. MicroRNAs play big roles in modulating macrophages response toward mycobacteria infection. Infect. Genet. Evol. 45, 378-382 (2016). 
16. Lin Y, Zhang Y, Yu H, Tian R, Wang G, Li F. Identification of unique key genes and miRNAs in latent tuberculosis infection by network analysis. Mol. Immunol. 112, 103-114 (2019).

17. Wang XW, Liu JJ, Wu QN, Wu SF, Hao DJ. The in vitro and in vivo effects of microRNA-133a on intervertebral disc destruction by targeting MMP9 in spinal tuberculosis. Life Sci. 188, 198-205 (2017).

18. Sahu SK, Kumar M, Chakraborty S et al. MicroRNA 26a (miR-26a)/KLF4 and CREB-C/EBPbeta regulate innate immune signaling, the polarization of macrophages and the trafficking of Mycobacterium tuberculosis to lysosomes during infection. PLoS Pathogens 13(5), e1006410 (2017).

19. Max KEA, Bertram K, Akat KM et al. Human plasma and serum extracellular small RNA reference profiles and their clinical utility. Proc. Natl Acad. Sci. USA 115(23), E5334-E5343 (2018).

20. Zhang X, Guo J, Fan SF et al. Screening and identification of six serum microRNAs as novel potential combination biomarkers for pulmonary tuberculosis diagnosis. PLoS ONE 8(12), (2013).

- Differentially expressed serum microRNAs (miRNAs) can be used as potential biomarkers for the diagnosis of pulmonary tuberculosis (TB).

21. Morris KV, Mattick JS. The rise of regulatory RNA. Nat. Rev. Genet. 15(6), 423-437 (2014).

22. Sana J, Faltejskova P, Svoboda M, Slaby O. Novel classes of non-coding RNAs and cancer. J. Transl. Med. 10, 103 (2012).

23. Aravin A, Gaidatzis D, Pfeffer S et al. A novel class of small RNAs bind to MILI protein in mouse testes. Nature 442(7099), 203-207 (2006).

24. Girard A, Sachidanandam R, Hannon GJ, Carmell MA. A germline-specific class of small RNAs binds mammalian Piwi proteins. Nature 442(7099), 199-202 (2006).

25. Grivna ST, Beyret E, Wang Z, Lin HF. A novel class of small RNAs in mouse spermatogenic cells. Genes Dev. 20(13), 1709-1714 (2006).

26. Kim VN. Small RNAs just got bigger: Piwi-interacting RNAs (piRNAs) in mammalian testes. Genes Dev. 20(15), 1993-1997 (2006).

27. Pek JW, Patil VS, Kai T. piRNA pathway and the potential processing site, the nuage, in the Drosophila germline. Dev. Growth Differ. 54(1), 66-77 (2012).

-. The primary processing pathway functions in both germline and somatic cells in the Drosophila ovaries. In contrast, the secondary processing pathway functions only in the germline cells.

28. Gu TT, Elgin SCR. Maternal depletion of Piwi, a component of the RNAi system, impacts heterochromatin formation in Drosophila. PLoS Genet. 9(9), e1003780 (2013).

- Failure of heterochromatin formation in the early embryo impacts the phenotype of the adult.

29. Hirano T, Hasuwa H, Siomi H. Identification of mouse piRNA pathway components using anti-MIWI2 antibodies. Methods Mol. Biol. 1463, 205-216 (2017).

30. Tang W, Tu S, Lee HC, Weng Z, Mello CC. The RNase PARN-1 trims piRNA $3^{\prime}$ ends to promote transcriptome surveillance in $C$. elegans. Cell 164(5), 974-984 (2016).

31. Weng C, Kosalka J, Berkyurek AC et al. The USTC co-opts an ancient machinery to drive piRNA transcription in C. elegans. Genes Dev. 33(1-2), 90-102 (2019).

32. Zhou X, Zuo Z, Zhou F et al. Profiling sex-specific piRNAs in zebrafish. Genetics 186(4), 1175-1185 (2010).

33. Varjak M, Donald CL, Mottram TJ et al. Characterization of the Zika virus induced small RNA response in Aedes aegypti cells. PLoS Neglected Trop. Dis. 11(10), e0006010 (2017).

34. Ninova M, Griffiths-Jones S, Ronshaugen M. Abundant expression of somatic transposon-derived piRNAs throughout Tribolium castaneum embryogenesis. Genome Biol. 18 (2017).

35. Goriaux C, Desset S, Renaud Y, Vaury C, Brasset E. Transcriptional properties and splicing of the flamenco piRNA cluster. Embo. Rep. 15(4), 411-418 (2014).

36. Klattenhoff C, Theurkauf W. Biogenesis and germline functions of piRNAs. Development 135(1), 3-9 (2008).

-. Reviews recent studies of piRNA production and function, and discuss unanswered questions about this intriguing new class of small RNAs.

37. Zhao K, Cheng S, Miao N et al. A Pandas complex adapted for piRNA-guided transcriptional silencing and heterochromatin formation. Nat. Cell Biol. doi:10.1038/s41556-019-0396-0 (2019).

38. Brennecke J, Aravin AA, Stark A et al. Discrete small RNA-generating loci as master regulators of transposon activity in Drosophila. Cell 128(6), 1089-1103 (2007)

39. Huang G, Hu H, Xue X et al. Altered expression of piRNAs and their relation with clinicopathologic features of breast cancer. Clin. Transl. Oncol. 15(7), 563-568 (2013).

40. Hashim A, Rizzo F, Marchese G et al. RNA sequencing identifies specific PIWI-interacting small non-coding RNA expression patterns in breast cancer. Oncotarget 5(20), 9901-9910 (2014). 
41. Rizzo F, Rinaldi A, Marchese G et al. Specific patterns of PIWI-interacting small noncoding RNA expression in dysplastic liver nodules and hepatocellular carcinoma. Oncotarget 7(34), 54650-54661 (2016).

42. Qiu W, Guo X, Lin X et al. Transcriptome-wide piRNA profiling in human brains of Alzheimer's disease. Neurobiol. Aging 57, 170-177 (2017).

43. Roy J, Sarkar A, Parida S, Ghosh Z, Mallick B. Small RNA sequencing revealed dysregulated piRNAs in Alzheimer's disease and their probable role in pathogenesis. Mol. Biosyst. 13(3), 565-576 (2017).

44. Busch J, Ralla B, Jung M et al. Piwi-interacting RNAs as novel prognostic markers in clear cell renal cell carcinomas. J. Exp. Clin. Canc. Res. 34, 61 (2015).

45. Muller S, Raulefs S, Bruns P et al. Next-generation sequencing reveals novel differentially regulated mRNAs, IncRNAs, miRNAs, sdRNAs and a piRNA in pancreatic cancer. Mol. Cancer 14, 94 (2015).

- Reports a novel lncRNA, sncRNA and mRNA signature of PDAC. In silico prediction of ncRNA targets allowed for assigning potential functions to differentially regulated RNAs.

46. Chu HY, Hui GY, Yuan L et al. Identification of novel piRNAs in bladder cancer. Cancer Lett. 356(2), 561-567 (2015).

47. Cui L, Lou YR, Zhang XJ et al. Detection of circulating tumor cells in peripheral blood from patients with gastric cancer using piRNAs as markers. Clin. Biochem. 44(13), 1050-1057 (2011).

48. Yang X, Cheng YD, Lu Q, Wei JF, Yang HW, Gu M. Detection of stably expressed piRNAs in human blood. Int. J. Clin. Exp. Med. 8(8), 13353-13358 (2015).

49. Xing Zhang MZ, Rong Yang, Weifeng Zhao, Xiaolong Hu, Gan AJ. Identification and comparison of novel circular RNAs with associated co-expression and competing endogenous RNA networks in pulmonary tuberculosis. Oncotarget 2(69), 113571-113582 (2017).

50. Zhang X, Zhu M, Hu X. Integrated miRNA and mRNA expression profiling to identify mRNA targets of dysregulated miRNAs in pulmonary tuberculosis. Epigenomics 10(8), 1051-1069 (2018).

51. Altschul SF, Gish W, Miller W, Myers EW, Lipman DJ. Basic local alignment search tool. J. Mol. Biol. 215(3), $403-410$ (1990).

52. Sun J, Wang SW, Li C, Ren YJ, Wang JQ. Novel expression profiles of microRNAs suggest that specific miRNAs regulate gene expression for the sexual maturation of female Schistosoma japonicum after pairing. Parasite Vector 7 (2014).

53. Liu B, Tian M, Guo Q et al. MiR-932 regulates pyrethroid resistance in Culex pipiens pallens (Diptera: Culicidae). J. Med. Entomol. doi:10.1093/jme/tjw083 (2016).

54. Bartel DP. MicroRNAs: target recognition and regulatory functions. Cell 136(2), 215-233 (2009).

55. Sasaki T, Shiohama A, Minoshima S, Shimizu N. Identification of eight members of the argonaute family in the human genome. Genomics 82(3), 323-330 (2003).

56. Juliano C, Wang J, Lin H. Uniting germline and stem cells: the function of Piwi proteins and the piRNA pathway in diverse organisms. Ann. Rev. Genet. 45, 447-469 (2011).

57. Guo M, Wu Y. Fighting an old war with a new weapon-silencing transposons by Piwi-interacting RNA. IUBMB life 65(9), 739-747 (2013).

58. Ku HY, Lin HF. PIWI proteins and their interactors in piRNA biogenesis, germline development and gene expression. Natl Sci. Rev. 1(2), 205-218 (2014).

- Provides evidence that PIWI proteins and piRNAs are not only crucial for transposon silencing in the germline, but also mediate novel mechanisms of epigenetic programming, DNA rearrangements, mRNA turnover and translational control both in the germline and in the soma.

59. Yin H, Lin HF. An epigenetic activation role of Piwi and a Piwi-associated piRNA in Drosophila melanogaster. Nature 450(7167), 304-U316 (2007).

60. Watanabe T, Lin HF. Posttranscriptional regulation of gene expression by Piwi proteins and piRNAs. Mol. Cell 56(1), 18-27 (2014).

61. Freedman JE, Gerstein M, Mick E et al. Diverse human extracellular RNAs are widely detected in human plasma. Nat. Commun. 7 , 11106 (2016).

- Presents comprehensive data to demonstrate the broad and consistent detection of diverse classes of circulating noncellular small-RNAs from a large population.

62. Zhang P, Kang JY, Gou LT et al. MIWI and piRNA-mediated cleavage of messenger RNAs in mouse testes. Cell Res. 25(2), 193-207 (2015).

63. Rouget C, Papin C, Boureux A et al. Maternal mRNA deadenylation and decay by the piRNA pathway in the early Drosophila embryo. Nature 467(7319), 1128-1132 (2010).

64. Weick EM, Miska EA. piRNAs: from biogenesis to function. Development 141(18), 3458-3471 (2014).

65. Siomi MC, Sato K, Pezic D, Aravin AA. PIWI-interacting small RNAs: the vanguard of genome defence. Nat. Rev. Mol. Cell Biol. 12(4), 246-258 (2011). 
66. Ishizu H, Iwasaki YW, Hirakata S et al. Somatic primary piRNA biogenesis driven by cis-acting RNA wlements and trans-Acting Yb. Cell Rep. 12(3), 429-440 (2015).

67. Jones BC, Wood JG, Chang C et al. A somatic piRNA pathway in the Drosophila fat body ensures metabolic homeostasis and normal lifespan. Nat. Commun. 7, 13856 (2016).

68. Russell S, Patel M, Gilchrist G et al. Bovine piRNA-like RNAs are associated with both transposable elements and mRNAs. Reproduction 153(3), 305-318 (2017). 